\title{
A dielectric-only superstrate inspired from transformation optics for complete reorientation of electromagnetic waves in azimuthal plane
}

\author{
Chetan Joshi*, Anne Claire Lepage, and Xavier Begaud \\ LTCI, CNRS, Télécom ParisTech, Université Paris-Saclay, 46 rue Barrault, 75013 Paris, France
}

Received 29 March 2016 / Accepted 1 June 2016

\begin{abstract}
Various applications require an antenna to provide coverage in the azimuthal plane while still retaining a low profile. Transformation Optics method can be used to provide novel solutions to fulfill this objective. In this paper, the design of dielectric superstrate inspired from Transformation Optics has been reported which completely reorients the electromagnetic waves emanating from a directive antenna in the azimuthal plane. The physical space is modeled using a virtual space composed of two opposed quarter circles. It is shown analytically that a dielectric-only solution can be obtained from the transformation relations. Use of shift and compression factors has been applied to the material parameter profile to achieve the reorientation. A full wave solution has been presented which reorients the radiation pattern of a classical patch antenna in the azimuthal plane. The solution can be fabricated from isotropic dielectric materials.
\end{abstract}

Key words: Antenna, Transformation Optics, Permittivity, Permeability, Refractive index, Radiation pattern.

\section{Introduction}

Today antennas need to fulfill increasingly difficult design specifications, especially in industries of aerospace and transport. Most applications require the antennas to maintain a low profile to minimize the effect of drag, while still emitting with a desired radiation pattern. More specifically, applications like telemetry, air-traffic control radar beacon, etc. require omnidirectional coverage in azimuthal plane. In elevation plane, this resembles an antipodal radiation pattern with the maxima in azimuthal plane and a null in broadside direction. The blade antenna is one of the most commonly held for such applications, owing to its low cost and simplicity in design [1]. Indeed, this comes at a compromise with the aerodynamic profile that can be an issue in vehicular platforms moving at high velocities. Emerging design techniques like Transformation Optics (TO) can provide an alternative [2, 3]. The premise of the TO concept is that the form of Maxwell's equations remains invariant under the coordinate transformation. The concept therefore allows to propose new material parameters (MP) of the transformed medium i.e. permittivity and permeability on the basis of coordinate transformation relating the physical space to a virtual space. TO has been used to

*e-mail: chetan.joshi@telecom-paristech.fr devise invisibility cloaks [4], illusion optics [5], compressed lenses [6] and other very interesting concepts [7, 8]. For radiating application, TO is used to modify the medium surrounding the source of electromagnetic waves i.e. antenna in order to change or improve the radiation properties of the device.

The definitive challenge of using Transformation Optics lies in the implementation of the MP profile as it contains spatially varying tensors of permittivity and permeability. Such MP profiles cannot be designed using natural materials. Anisotropy of such profiles has often been achieved using split ring resonators (SRR) type of metamaterials [9]. This design technique forms the basis to achieve anisotropy in MP profile in a variety of proposed solutions [4, 10-14]. However, the inherently dispersive nature of the SRR makes the design lossy and narrowband. Therefore this method is not ideal to design TO devices, especially for antenna applications. The challenge in prototyping of TO devices with SRR technology is more daunting when confronted with extreme material parameters i.e. extremely high, near zero or negative values of permittivity and permeability. Hence, simpler approaches are sought to design these devices.

A more convenient way to prototype TO devices is to design a dielectric-only MP profile. Under appropriate design conditions, the contribution of permeability values in profile can be ignored and it may be interpreted using only 
permittivity values [15-17]. A purely dielectric profile is much easier to realize in fabrication using either electric-field-driven LC (ELC) resonator structures [18-22] or using isotropic standard dielectric materials [23]. An ELC resonator is a metallic pattern lithographically printed on a substrate that is capable of providing a strong electric response. ELC resonators are used to achieve permittivity values, $\varepsilon<1$ that cannot be achieved using natural materials $[18,19]$. However, other practical realizations of dielectric TO devices ignore these values $[20,21,23]$. Dielectric-TO solutions with isotropic permittivity present a great advantage when designing Graded Refractive Index (GRIN) type of devices using standard dielectric materials [23-27]. These designs offer the advantage of broadband frequency operation [15]. Various design approaches have been used to achieve such MP profiles e.g. perforated dielectric [23], micro and nano-sized powders of processed titanates [24], etc. Also, the 3D printing techniques may be used to prototype such devices easily.

More specifically on the use of TO for antennas, radiation properties of the antenna can be improved or modified by using TO devices [11-14, 16, 17, 19, 22, 24-27]. Kong presents a focusing antenna, using TO to flatten the parabolic reflector [11]. Tichit reports highly directive radiation obtained using TO-based lenses [12]. It uses metamaterials to achieve the anisotropic permittivity and permeability. Metamaterial based and dielectric-only solutions have been reported to steer the main lobe of the antenna [19, 25, 27]. TO has also been used to design an all dielectric superstrate that restores in-phase emissions from radiating elements conformal to a cylindrical surface such as fuselage of an aircraft [26]. Wu reported use of TO to design multi beam lenses [13]. Depending upon the chosen transformation, the lenses can be used to provide multiple collimated beams in the azimuthal plane of a monopole antenna. This allows to achieve the desired antipodal pattern in a two dimensional plane. In [13], quarter wavelength monopole has been used as a feeding source and the TO lens surrounding it needs to be at least as thick as the height the monopole. The actual reported thickness of the collimating lens is in the order of half-wavelength at lowest frequency of operation. Such thickness may be impractical, certainly as we look for solutions in lower frequency ranges. Hence an alternative approach to achieve the antipodal radiation pattern is needed. In a previous work, we have introduced a concept where a TO superstrate was placed over a patch antenna to achieve the antipodal radiation pattern [14]. The design complexity of this device was extremely high due to anisotropic nature of MP profile, to a very large variation in the permittivity $\left(0<\varepsilon_{\mathrm{zz}}<19.7\right)$ and permeability $\left(0<\mu_{\mathrm{yy}}<25\right)$ values. Indeed, extreme material parameters i.e. $\mu, \varepsilon<1$ also show large spatial presence in the profile, which demands that such material parameters be taken in account in the discretized solution.

In this paper, we shall present a dielectric superstrate to completely reorient the electromagnetic waves in the azimuthal plane of a planar source antenna. In Section 2, the problem will be studied in two dimensions to reduce design complexity. First, simple mathematical constructions of the physical and virtual spaces will be defined. Then, expressions of constitutive parameters to interpret the transformed spaces will be derived using TO method. A shift factor will be introduced to shift the refractive index profile. It will be shown that a dielectric-only reinterpretation of the device is possible. Next, axial and lateral compression will be introduced in the transformation, which shall allow designing a small and low-profile superstrate. In Section 3, a full wave solution will be designed. First, the antenna used as source will be described briefly. Then, continuously varying dielectric profile designed in Section 2 will be discretized into a finite number of pixels. This discretized profile will be then extruded to obtain a cuboidal superstrate. Use of a matching layer to match the combination of superstrate and antenna at the input will be discussed. The solution reported in this paper clearly demonstrates the advantage in terms of reduced parameter complexity, low profile and reorientation of electromagnetic waves.

\section{Two dimensional modeling of problem}

A TO superstrate is desired such that when placed over a patch antenna, the electric field is reoriented in the azimuthal plane of the antenna (Figure 1). In this section, transformation relations between the physical and virtual spaces in $x-y$ plane of the Cartesian unprimed space are considered, and the profiles are kept untransformed along $z$-axis. The problem has been simulated with COMSOL Multiphysics design suite [28]. All the solutions are calculated at a frequency chosen in L-band, $f=1.25 \mathrm{GHz}$. The transformation optics method gives MP values, which can be defined with COMSOL. The patch antenna is modeled using an idealized current sheet backed with a PEC plane, which provides a $z$-component of electric field only. This permits reducing dependence on only few elements of tensors in the solution. This has been discussed later in this section. Finally, all solutions are calculated in a circular domain. For a better simulation of the far field performance of the superstrate, absorbing boundary conditions have been applied in the far field region of the antenna. In the first sub-section, a mathematical construction of the physical and virtual spaces has been defined.

\subsection{Description of physical and virtual spaces}

As said in the problem statement in the beginning of Section 2, it is desired that the transformed space completely reorient the electric field in the azimuthal plane of the antenna. To achieve this reorientation using TO concept, an appropriate virtual space needs to be defined. In [14], two quarter ellipses have been used to construct the virtual space. A similar construction of virtual space has been used in present work, as described below.

The physical space is defined in an unprimed coordinate system $(x, y, z)$. The virtual space is defined in a primed coordinate system $\left(x^{\prime}, y^{\prime}, z^{\prime}\right)$. The physical space can be thought as a rectangle of length $2 \times e$ and width $d$, as shown in Figure 2a. The virtual space can be envisioned as two opposed quarter circles as shown in Figure 2b, where $l$ and $e$ represent the size of the virtual space in unprimed coordinate system. The transformation relation to transform blue lines of Figure 2 is given in equation (1). The red lines are transformed using 


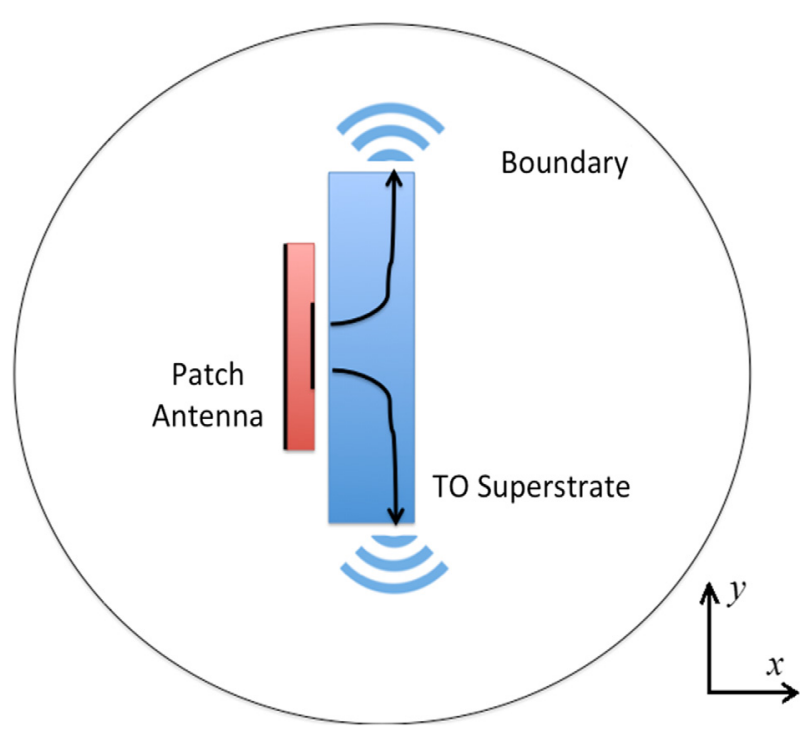

Figure 1. Representative diagram of the problem, the superstrate reorients the electromagnetic waves in the azimuthal plane of the patch antenna. (a)

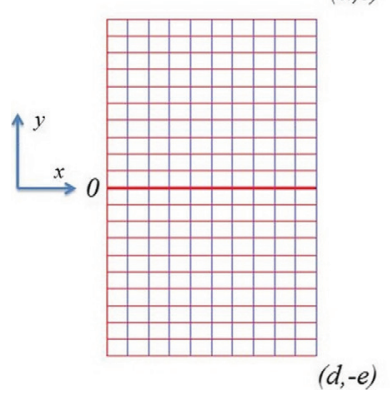

(b)

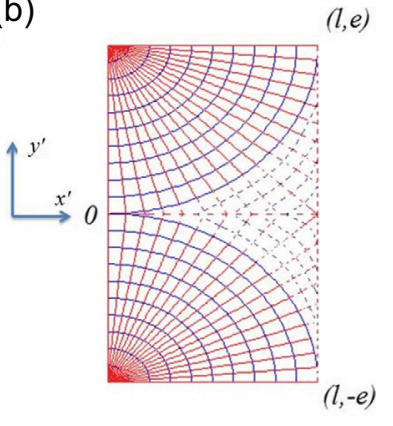

Figure 2. (a) Physical space, (b) virtual space.

equation (2). These two equations are used to generate the loci along which the field shall be reoriented. It is important to note that both the equations are independent of $d$ and $l$, and that these two variables have been used to demarcate the physical and virtual space. It is also important to note that in Figure $2 b$ desired propagation path for complete reorientation along $y$-axis has been emphasized using the grid generated in solid lines. However, transformation relations also generate a grid in the remaining region as shown using dashed lines. Therefore, transformation does not prohibit propagation along $x$.

$$
\begin{aligned}
& x^{\prime}=\sqrt{(x)^{2}+(y \pm e)^{2}} \\
& y^{\prime}=\frac{e}{\pi} \tan ^{-1}\left(\frac{y-e}{x}\right)
\end{aligned}
$$

The Jacobian matrix relating the physical and virtual spaces can be calculated. It is given in equation (3).

$$
J=\left[\begin{array}{ccc}
\frac{x}{\sqrt{(x)^{2}+(y \pm e)^{2}}} & \frac{y \pm e}{\sqrt{(x)^{2}+(y \pm e)^{2}}} & 0 \\
\frac{-e}{\pi} \frac{(y \pm e)}{(x)^{2}+(y \pm e)^{2}} & \frac{e}{\pi} \frac{x}{(x)^{2}+(y \pm e)^{2}} & 0 \\
0 & 0 & 1
\end{array}\right]
$$

As the profile remains unchanged in $z$-axis, the $\frac{\partial z^{\prime}}{\partial z}=1$ in equation (3). Using TO method given in [2], the Jacobian matrix can now be used to find the constitutive parameter profiles that link the physical and virtual spaces. In the present work, the derived expressions represent the relative permittivity and permeability. This is shown in equation (4). Thus, the required transformation may be achieved using this profile.

$$
\mu^{\prime}=\varepsilon^{\prime}=\left[\begin{array}{ccc}
\frac{\pi x^{\prime}}{e} & 0 & 0 \\
0 & \frac{e}{\pi x^{\prime}} & 0 \\
0 & 0 & \frac{\pi x^{\prime}}{e}
\end{array}\right]
$$

It is seen in equation (4), that the transformation of space can be realized using the six elements given by the diagonal elements of the two matrices. Indeed, as only $E_{\mathrm{z}}$ is considered at the input, the material parameter dependence can be reduced to only three material parameters: $\mu_{\mathrm{xx}}, \mu_{\mathrm{yy}}$ and $\varepsilon_{\mathrm{zz}}$ which are defined in equation (5).

$$
\mu_{\mathrm{xx}}^{\prime}=\frac{\pi x^{\prime}}{\mathrm{e}} ; \mu_{\mathrm{yy}}^{\prime}=\frac{1}{\mu_{\mathrm{xx}}^{\prime}} ; \varepsilon_{\mathrm{zz}}^{\prime}=\frac{\pi x^{\prime}}{\mathrm{e}}
$$

With COMSOL, the rectangular physical space is defined such that $d=e=l=\lambda / 2$, where $\lambda$ represents the wavelength at $f$, i.e. $\lambda=24 \mathrm{~cm}$. In Figure $3, E_{z}$ has been plotted in the calculation domain; the plots are normalized to the maximum value of the electric field. It is seen that electric field has been partially reoriented in the azimuthal plane of the antenna along $y$-axis. The corresponding material parameters have been plotted in Figure 4. The distribution of the material parameters along equation (1) and equation (2) can be clearly noticed. These results validate the hypothesis that chosen transformations can reorient the electric field in the azimuthal plane. As mentioned earlier, the physical space is not sufficiently transformed so as to completely reorient the electric field along $y$. The grid generated in the dashed region of Figure $2 \mathrm{~b}$ allows propagation of $E_{z}$ along $x$-axis as well. This may be attributed to the finite size of the transformed space. However, additional steps can always be undertaken to exert more control. This is discussed in more detail in the next sub sections.

\subsection{Refractive index based interpretation and shift factor " $m$ "}

In the previous subsection, equation (5) gives the variation in the permittivity and permeability values in the transformed profile. These values can be used to define the variation of effective refractive index, $n_{\text {eff }}$ in the modified space. In equation (6), $n_{\mathrm{eff}}$ has been interpreted using values from 


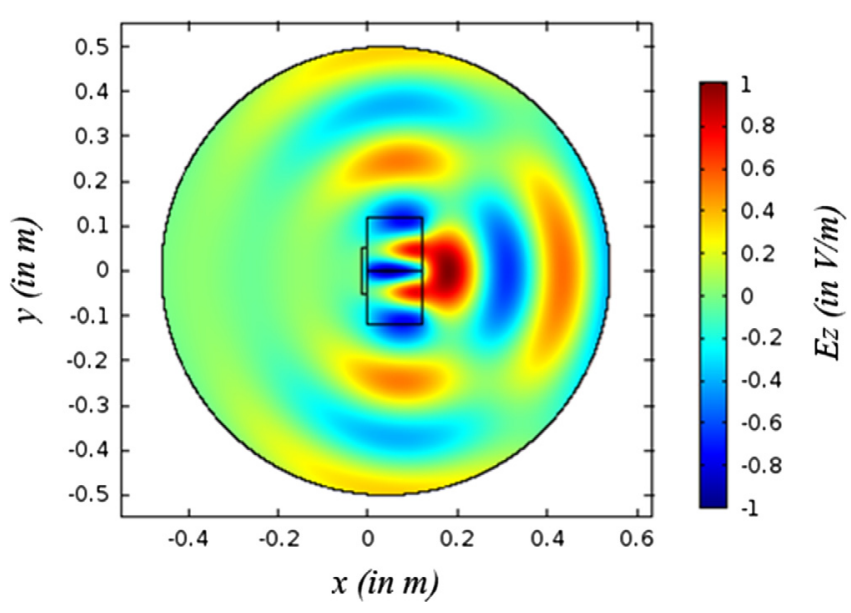

Figure 3. Partially reoriented electric field shows that chosen transformation relations can achieve the desired reorientation.

equation (5). A new factor ' $m$ ' has also been introduced that can be used to shift the refractive index profile.

$$
n_{e f f}=m \sqrt{\mu_{x x}^{\prime} \varepsilon_{z z}^{\prime}} \sqrt{\mu_{y y}^{\prime} \varepsilon_{z z}^{\prime}}
$$

On multiplication by $m$, only the value of refractive indices in the profile is changed but the new $n_{\text {eff }}$ continues to vary along the previously defined loci. This factor can be used as an additional degree of freedom to control the radiation pattern and to eliminate extremely high or low values of material parameters. $m$ shall be used later to shift the refractive index profile compensate for the changes arising due to compression. This has been discussed later in this Section 2.3.

One of the major problems in fabrication of TO device is the anisotropy in the solution. This is also the case in present case, as seen in equation (5). In [14], a simplification of MP profile was proposed to limit variation to two MP's $\left(\mu_{\mathrm{yy}}\right.$ and $\left.\varepsilon_{\mathrm{zz}}\right)$ to facilitate a SRR-type metamaterial solution. The material parameters achieved from this simplification present a significant challenge in realization due to low and high anisotropic values of permeability and permittivity. Li has presented a strategy to realize TO solutions using isotropic dielectrics [15]. It is reported that transformations can be generated such that the physical space is perceived as a virtual space of an isotropic homogenous medium permittivity and $\mu_{\mathrm{r}}=1$. Tang has shown that for $E_{\mathrm{z}}$ polarized field, the magnetic dependence can be removed if $\left(\mu_{\mathrm{xx}} \cdot \mu_{\mathrm{yy}}\right)=1$, and interpretation using only dielectric materials can approximate the refractive indices of the transformed profile [16].

It may be noted in equation (5) that $\mu_{\mathrm{xx}}$ and $\mu_{\mathrm{yy}}$ are reciprocal values. This allows $n_{\text {eff }}$ to be mapped out using only permittivity tensor in equation (6) i.e. $\varepsilon_{\mathrm{zz}}$, and permeability values can be ignored. Thus, the chosen transformation relations indicate that the root profile defined using transformation relations (1) and (2) allows a dielectric-only solution. This presents an opportunity to further simplify the profile. This simplification is used in the next subsection to design a dielectric-only profile. Additionally, it is required to achieve the desired reorientation in a low profile device. This can be achieved by applying compression on physical and virtual space. This has also been discussed below.

\subsection{Dielectric superstrate with lateral and axial compression}

In Section 2.1, the physical space has dimensions of $\lambda \times 0.5 \lambda$. Such a solution is bulky and impractical. It is desired to achieve the reorientation of electric field in a small and lowprofile space. Indeed, the principle of TO can be used once again to introduce different compressions in the design. Let us reconsider (1) and (2), which transformed a rectangular space into two opposed quarter circles. Now, compression is introduced in the modified space such that $n_{\text {eff }}$ varies along compressed elliptical loci as shown in Figure 5. The modified transformation relations are given by (7) and (8). Two different compressions are introduced: axial compression " $a$ " and lateral compression factor " $b$ ".

$$
\begin{gathered}
x^{\prime}=\sqrt{(a x)^{2}+(b(y \pm e))^{2}} \\
y^{\prime}=\frac{e}{\pi} \tan ^{-1}\left(\frac{b(y-e)}{a x}\right)
\end{gathered}
$$

The new material parameters can be calculated using the TO method as shown earlier. As seen in the last sub-section, the underlying transformation can be interpreted using dielectric materials only. Therefore, we assume in this section that the space transformed by introducing compression factors can be interpreted using dielectric material parameters only i.e. $\varepsilon^{\prime}{ }_{\mathrm{zz}}$ will be considered only, while $\mu_{\mathrm{xx}}=\mu_{\mathrm{yy}}=1$. The new expression of permittivity, $\varepsilon_{z z}^{\prime}$ has been given in equation (9).

$$
\varepsilon_{z z}^{\prime}=\frac{m \pi x^{\prime}}{a b e}
$$

It may be noted here, that the previously defined shift factor $m$ has been directly introduced in the expression of permittivity tensor. When the profile is compressed by $a$ along $x$ and by $b$ along $y$, the MP profile registers an expansion along $z$. This expansion is seen by the presence of $a \times b$ in the denominator of equation (9). In other words, compression along $x$ and $y$ leads to rarefaction or dilution of the $z z$ tensor component by a factor of $a \times b$. Consequently, this also has

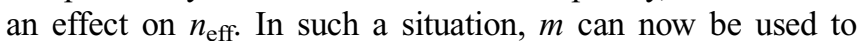
adjust the value of $n_{\text {eff }}$ to achieve the desired reorientation. Thus, the final solution can be considered as interplay of three factors: $a, b$ and $m$.

First the compression is introduced in the problem, while $m=1$. In the current problem, compression factors are chosen as $a=4.2$ and $b=1.6$. This leads to new dimension of physical and virtual space i.e. $d=l=0.12 \lambda, e=0.3125 \lambda$. Therefore, this newly transformed space has dimensions of $0.625 \lambda \times 0.12 \lambda$. This is a considerable reduction in the size 

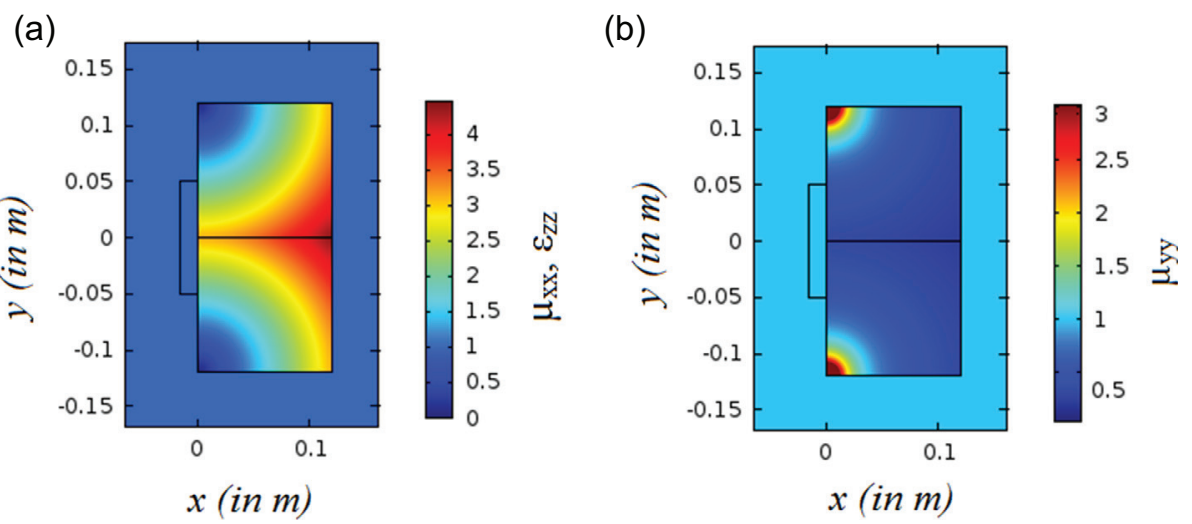

Figure 4. Variation of material parameters in transformed profile (a) $\mu_{\mathrm{xx}}$ and $\varepsilon_{\mathrm{zz}}$, (b) $\mu_{\mathrm{yy}}$.

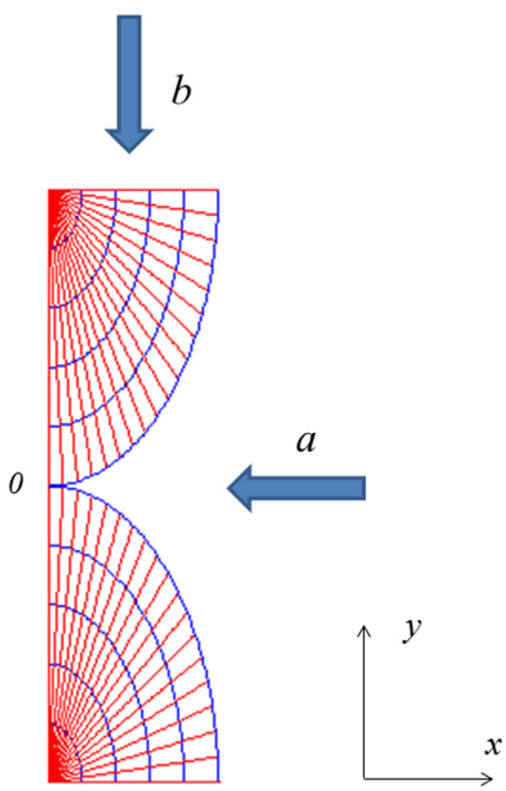

Figure 5. Compressed space based on new transformation relations.

of the superstrate. The new dielectric profile varies along the elliptical loci. But, the expansion in $z z$ element of tensor owing to compression in equation (9) leads to a rarified profile with respect to equation (5). Such a profile is incapable of reorienting $E_{z}$ in the azimuthal plane. Now, a parametric simulation is run on $m$ to optimize the profile for complete reorientation. For $m=14$, the $E$ field is found to be guided completely along $y$-axis, as seen in Figure 6. In Figure 7, the time averaged power flow at the boundary has been plotted for $m=1$ and 14. It clearly shows how $m$ can be used to correct the expansion in the profile arising due to compression factors. The power flow in the broadside $\left(0^{\circ}\right)$ is $20 \mathrm{~dB}$ less than in the azimuthal plane $\left( \pm 90^{\circ}\right)$. As the boundary conditions lie in the far-field region, the power flow diagram gives a quasiapproximation of the far field pattern. Other combinations of $a, b$ and $m$ may also provide reorientation in the profile. However, this may come at the expense of increase in size.

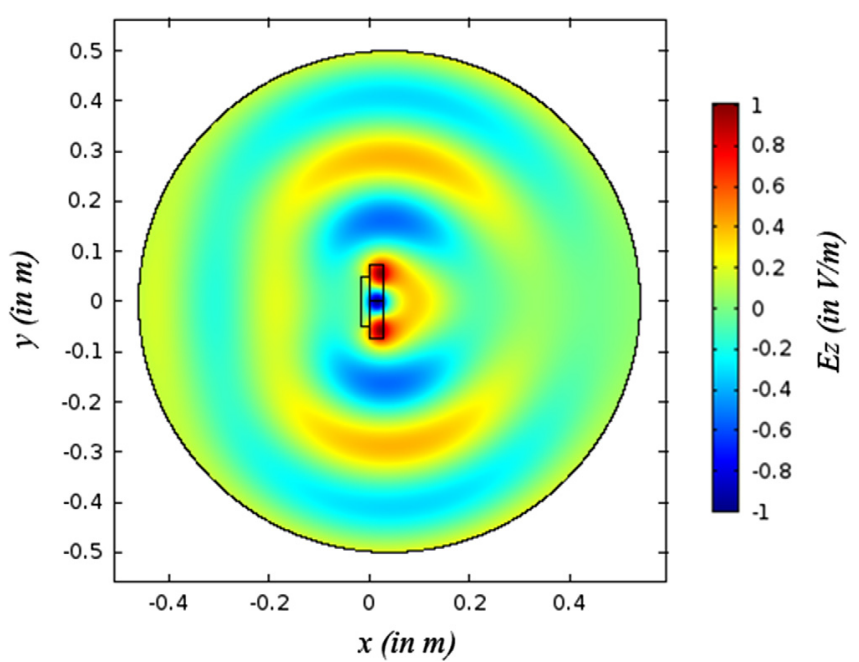

Figure 6. Completely reoriented electric field in the compressed dielectric profile with the shifted refractive index profile.

The permittivity varies between $0<\varepsilon_{\mathrm{zz}}<14.8$. In Figure 8 , the permittivity variation has been plotted in the range $1<\varepsilon_{\mathrm{zz}}<14.8$. Thanks to $m$, the spatial presence of permittivity values $\varepsilon<1$ has been limited to a very small region, as represented by the uncolored region at the corners of the profile. Therefore, these values can be conveniently ignored in the ensuing design steps. In the next section, a full wave solution shall be discussed. The dielectric profile designed in this section shall be used to arrive at a practical solution.

\section{Full wave solution}

In the previous section, a small low profile space has been designed to completely reorient the electromagnetic waves emanating from an antenna in the azimuthal plane. It may be recalled that a patch antenna was modeled using an idealized current sheet backed with a PEC plane. Design issues like mismatch at the input port or the antenna-superstrate interface 


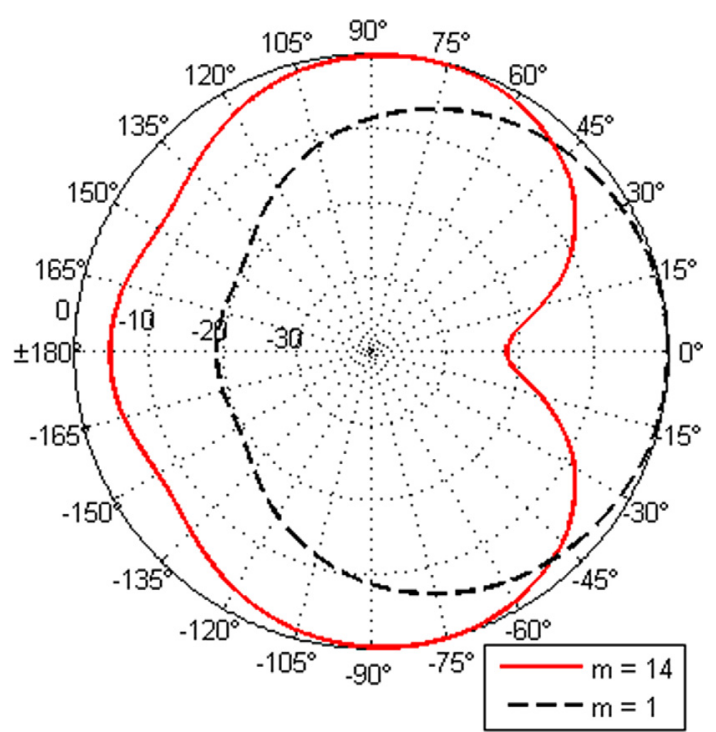

Figure 7. Time averaged power flow (in $\mathrm{dB}$ ) at the boundary; $m=1,14$.

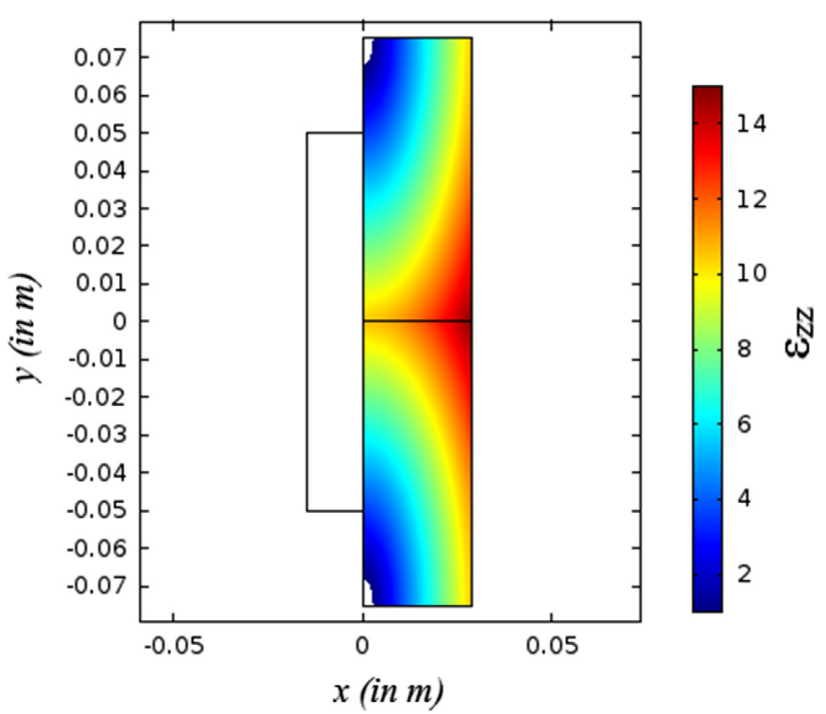

Figure 8. Dielectric-only material profile, $1<\varepsilon_{\mathrm{zz}}<14.8$.

had not been considered. In practice, an antenna is far from a canonical source, which is why a full wave solution needs to be considered. Also, the profile described in Section 2 consisted of continuously varying material parameters. In this section, a real patch antenna has been used to feed the superstrate. In the following sub-sections, the antenna will be presented; a material profile of Section 2 will be discretized to achieve finite number of pixels and the discretized profile will be extruded along $z$ to achieve a cuboidal superstrate. Use of a dielectric layer to match the antenna-superstrate assembly at input has also been discussed. Finally, the performance of the antenna with superstrate will be shown. All solutions have been calculated with transient solver in CST Microwave Studio [29].

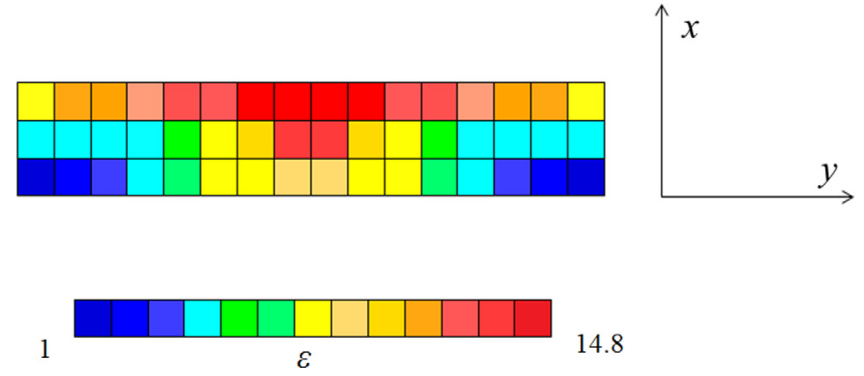

Figure 9. Material profile discretized in 48 pixels.

\subsection{Square patch antenna}

A classic square patch antenna has been considered in this study. The antenna is designed to operate at the frequency of the design, $f=1.25 \mathrm{GHz}$. The side of the square patch is $68 \mathrm{~mm}$. The antenna substrate is $13 \mathrm{~mm}$ thick and has a relative dielectric constant of $\varepsilon_{r}=2.5$. The patch antenna is backed by a square ground plane of side $110 \mathrm{~mm}$. Also, the antenna is fed using a coaxial feed. The antenna radiates with a broadside radiation pattern. This antenna shall be considered throughout this study. In the next subsection, the design of three dimensional superstrate has been discussed.

\subsection{Discretization of profile and 3D superstrate}

In Section 2.3, a continuously varying dielectric profile was obtained from TO method that completely reoriented the electric field along the $y$-axis. In this subsection, a three dimensional superstrate is obtained from that profile. It is now desired to arrive at an equivalent profile with finite number of pixels that may adequately represent the profile designed earlier. It is assumed that the dielectric profile is isotropic. Also, the values of $\varepsilon<1$ are discarded and replaced by $\varepsilon=1$. The profile is discretized into 48 pixels arranged in three layers of 16 pixels each, as shown in Figure 9. The chosen discretization allows envisaging a fabrication strategy using perforated dielectrics to achieve the gradient in the dielectric permittivity. This approach has been previously adopted in [27]. Higher values in the profile of $\varepsilon$ may be achieved using ceramic pins of varying diameters to fill up air holes.

It is intended to keep the profile untransformed along $z$ to respect the transformation. However, a finite size needs to be defined for the superstrate. Therefore, the profile is extruded along $z$ such that the final size of the superstrate is $0.625 \lambda \times 0.625 \lambda \times 0.12 \lambda$, as shown in Figure 10. In the next sub-section, the superstrate and the antenna will be studied together. Also, the use of a matching layer will be described.

\subsection{Superstrate with antenna}

In this section, the study of the superstrate coupled with antenna is presented. As seen in the previous sub-sections, a patch antenna has been designed to be used as a source and 


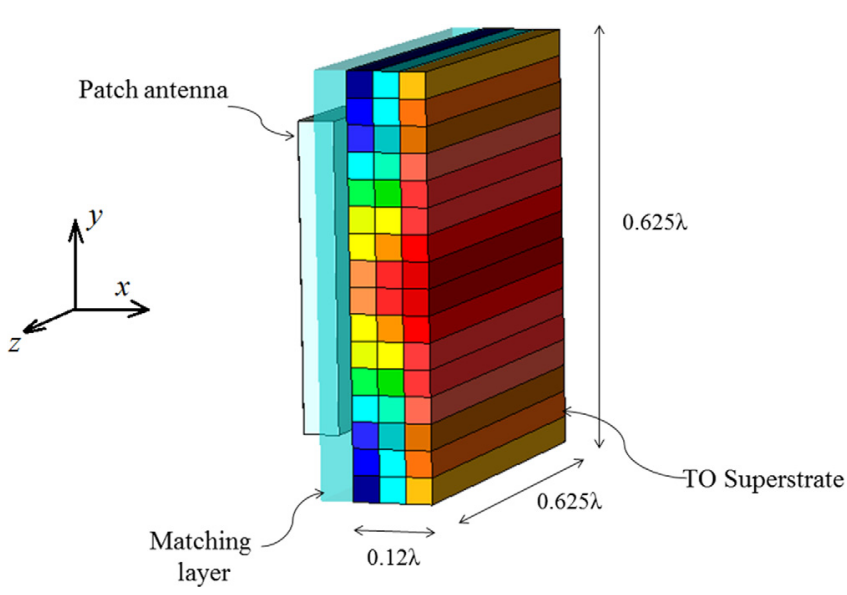

Figure 10. Antenna with the three dimensional superstrate, matching layer used for matching impedance at input.

a cuboidal superstrate has been designed with finite values of isotropic dielectric materials. The far field pattern of the patch antenna alone shows radiation in broadside direction, as shown in Figure 11a. It is expected that the field can be oriented in the azimuthal plane of the antenna by simply placing the superstrate above it.

However, when the two devices are combined together, the superstrate does not show the reorientation of field. Upon investigation, it is found out that the antenna fails to couple energy into the superstrate at the interface at the design frequency, $f$. Indeed, this is observed in Figure 12, where the reflection coefficient plotted in dashed orange line does not show any sharp resonance in the simulated frequency range. In order to achieve the coupling between the two devices, a matching layer of $10 \mathrm{~mm}$ is introduced between the antenna and the superstrate. In this case, matching layer is comprised of air. The new design is matched at new frequency $f^{\prime}=1.33 \mathrm{GHz}$, as seen in the dashed green line of Figure 12 . The electromagnetic energy is coupled from antenna to the superstrate. It was verified that the superstrate reorients the electric field in the entire operational bandwidth. It should be noted here that the size of individual pixels was adapted to $f^{\prime}$ by transposition of frequency, which is necessary to conserve the antipodal radiation pattern.

However, field needs to be reoriented at $f$. In order to shift the operation frequency from $f^{\prime}$ to $f$, a dielectric material is introduced in the gap between the antenna and the superstrate. A parametric simulation is run on the relative permittivity of this material. The antenna-superstrate assembly is matched at $f$ when $\varepsilon_{r}=1.8$. It may be noted that the size of individual pixels in the superstrate corresponds to $f$. The thickness of the dielectric material can be re-optimized to efficiently match the antenna at the input. The antenna is found to be matched when a $12 \mathrm{~mm}$ thick dielectric material is used in the matching layer. In this case the antenna presents a bandwidth (where magnitude of the input reflection coefficient is less than $-10 \mathrm{~dB})$ of $50 \mathrm{MHz}(4 \%$ at $1.25 \mathrm{GHz})$. The antenna reorients the electromagnetic waves along $y$, as shown in Figure 11b. In Figure 12, the reflection coefficient of the antenna superstrate assembly is plotted in the solid red line.

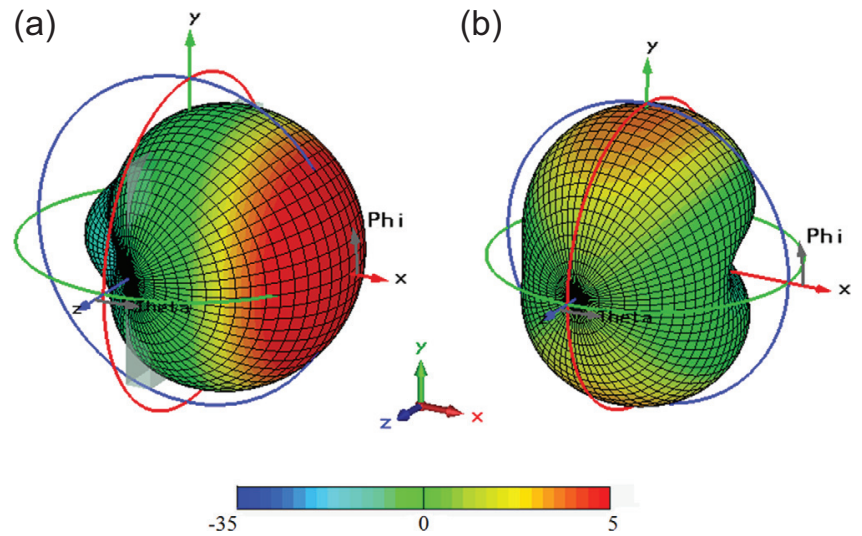

Figure 11. Far field directivity plots (in dBi): (a) Antenna alone with broadside radiation pattern, (b) Superstrate above antenna, antipodal radiation pattern in azimuthal plane.

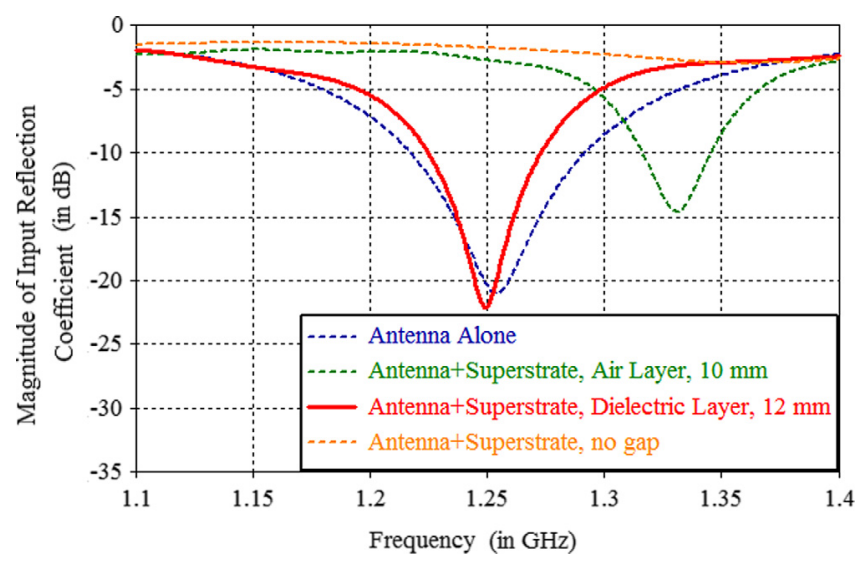

Figure 12. Magnitude of the input reflection coefficient for different antenna configurations.

In Figure 13, the far field pattern of the antenna with superstrate has been compared to the antenna without superstrate. Directivity has been plotted in the elevation plane. When compared with the directivity of the patch antenna, it is clearly visible that the superstrate reorients the radiation in the azimuthal plane of the antenna. Maximum directivity is $3.8 \mathrm{dBi}$ at $\pm 90^{\circ}$. Also, the reorientation is observed at all frequencies in the bandwidth of the device. The matching layer only improves power coupled from antenna to the superstrate, thus improving the matching at input of the antenna. It does not contribute to the reorientation of electric field in any significant manner. The drawback of using the matching layer is the increase in overall thickness of the superstrate from $0.12 \lambda$ to $0.17 \lambda$.

Thus, the superstrate type device reorients the electromagnetic waves emanating from a practical patch antenna. The performance of the device can be further optimized. The analysis of coarser or finer discretization is an interesting study for this transformation. In Section 3.2, the discretization of 48 pixels was chosen arbitrarily. Other pixel distributions can also be tested, depending upon the resolution desired. However it is outside the context of this present article. Also, the discretized profile was extruded along $z$ to design the cuboidal superstrate. 


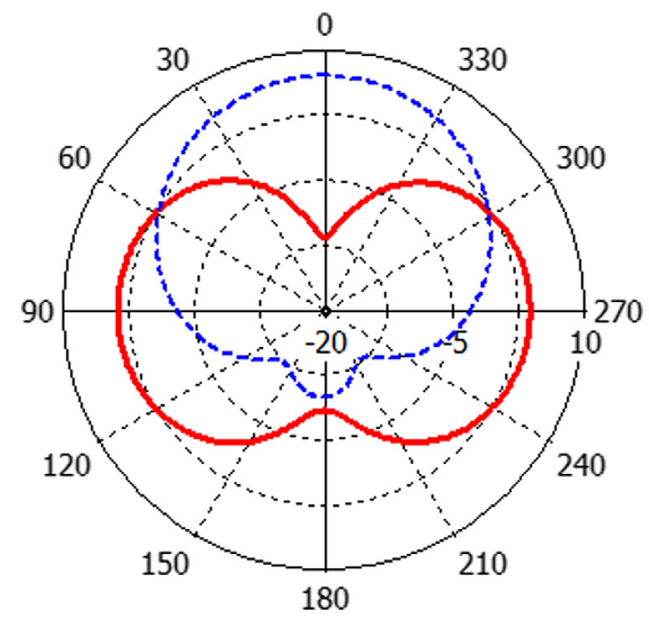

..... Antenna Alone

- Antenna Superstrate with Dielectric Layer, $12 \mathrm{~mm}$

Figure 13. Directivity (dBi) at $1.25 \mathrm{GHz}$.

Indeed, the profile can also be revolved about $x$-axis to give rise to a cylindrical device.

\section{Concluding remarks and perspectives}

In this paper, a superstrate was presented with a dielectriconly construction. The device was inspired from the TO concept. The transformation relations between physical and virtual spaces were defined and the expressions of constitutive material parameters were derived using TO method. A shift factor was also introduced that allowed to control the effective refractive index in the transformed profile, giving an additional degree of freedom. It was seen that the chosen transformation relations allowed a dielectric only interpretation of the transformation. Drawing from the above inferences, a compact, low profile superstrate was designed. Axial and lateral compression was applied on the physical and virtual spaces. Interplay between the compression factors and the previously defined shift factor was used to arrive at a profile that provided complete reorientation of electromagnetic waves in the azimuthal plane. This profile was then discretized into arbitrarily chosen number of pixels and was extruded to give rise to a three dimensional cuboidal superstrate. A practical patch antenna was used to feed this superstrate. It was seen that a matching layer was necessary between the antenna and superstrate to improve the coupling of power from antenna to superstrate. Also, a dielectric material was used as matching layer in order to shift the frequency of operation of the device. The superstrate showed reorientation of electromagnetic waves at all frequencies in the bandwidth. The study leads to an important conclusion that the antipodal radiation pattern may be obtained in a compact low profile device that can be made using standard dielectric materials.

One of the major obstacles towards fabrication of the superstrate presented in this study is the presence of high permittivity values. Various strategies may be adopted to fabricate such a superstrate. Micro and nano sized powderedtitanates have been used previously to achieve the high dielectric values. Also, drilled dielectric with alumina stems may be used realize the gradient in the refractive index. The design can be frequency-shifted to a frequency where materials with high dielectric permittivity are available. Furthermore, using the interplay of compression and shift factors, other values of permittivity can be envisaged, but which may come at the expense of increase in size of the device.

Acknowledgements. This work is supported by the NanoDesign project funded by IDEX Paris-Saclay, ANR-11-IDEX-0003-02.

\section{References}

1. T. Macnamara, in Introduction to antenna placement and installation, Chap. 2 and 5, John Wiley \& Sons Ltd., London, 2010.

2. J.B. Pendry, D. Schurig, D.R. Smith, Controlling electromagnetic fields, Science 312 (2006) 1780.

3. U. Leonhardt, Optical conformal mapping, Science 312 (2006) 1777.

4. D. Schurig, J.J. Mock, B.J. Justice, S.A. Cummer, J.B. Pendry, A.F. Starr, D.R. Smith, Metamaterial electromagnetic cloak at microwave frequencies, Science 314 (2006) 977.

5. Y. Lai, J. Ng, H.Y. Chen, D.Z. Han, J.J. Xiao, Z.Q. Zhang, C.T. Chan, Illusion optics: the optical transformation of an object into another object, Phys. Rev. Lett. 102 (2009) 253902.

6. D.A. Roberts, N. Kundtz, D.R. Smith, Optical lens compression via transformation optics, Optics Express 17 (2009) 16535.

7. D.H. Kwon, D.H. Werner, Transformation optics: an overview of the theory and applications, IEEE Antennas Propag. Mag. 52 (2010) 24.

8. W.X. Jiang, W.X. Tang, T.J. Cui, Transformation optics and applications in microwave frequencies, Progress Electromagn. Res. 149 (2014) 251.

9. W.X. Jiang, J.Y. Chin, T.J. Cui, Anisotropic metamaterial devices, Mater. Today 12 (2009) 26.

10. M. Rahm, D.A. Roberts, J.B. Pendry, D.R. Smith, Transformation-optical design of adaptive beam bends and beam expanders, Optics Express 16 (2008) 11555.

11. F.M. Kong, B.I. Wu, J.A. Kong, J.T. Huangfu, S. Xi, H.S. Chen, Planar focusing antenna design by using coordinate transformation technology, Appl. Phys. Lett. 91 (2007) 253509.

12. P.H. Tichit, S.N. Burokur, A. De Lustrac, Design and experimental demonstration of a high-directive emission with optical transformations, Phys. Rev. B 83 (2011) 155108.

13. Q. Wu, Z.H. Jiang, O. Quevedo-Teruel, J.P. Turpin, W. Tang, Y. Hao, D.H. Werner, Transformation optics inspired multibeam lens antennas for broadband directive radiation, IEEE Trans. Antennas Propag. 61 (2013) 5910.

14. M. Clemente Arenas, A.C. Lepage, X. Begaud, Antipodal radiation pattern of a patch antenna combined with superstrate using transformation electromagnetics, Appl. Phys. A 117 (2014) 699.

15. J. Li, J.B. Pendry, Hiding under the carpet: a new strategy for cloaking, Phys. Rev. Lett. 101 (2008) 203901. 
16. W. Tang, C. Argyropoulos, E. Kallos, W. Song, Y. Hao, Discrete coordinate transformation for designing all-dielectric flat antennas, IEEE Trans. Antennas Propag. 58 (2010) 3795.

17. W. Tang, Y. Hao, Transformation electromagnetics and metamaterials, Chap. 7, in D.H. Werner, D.H. Kwon (Eds.), Springer, London, 2014

18. D. Schurig, J.J. Mock, D.R. Smith, Electric-field-coupled resonators for negative permittivity metamaterials, Appl. Phys. Lett. 88 (2006) 041109.

19. J. Yi, S.N. Burokur, A. de Lustrac, Experimental validation of a transformation optics based lens for beam steering, Appl. Phys. Lett. 107 (2015) 154101.

20. H. Ma, W. Jiang, X. Yang, X. Zhou, T. Cui, Compact-sized and broadband carpet cloak and free-space cloak, Optics Express 17 (2009) 19947.

21. N. Kundtz, D.R. Smith, Extreme-angle broadband metamaterial lens, Nature Mater. 9 (2010) 129.

22. P.H. Tichit, S.N. Burokur, C.-W. Qiu, A. de Lustrac, Experimental verification of isotropic radiation from a coherent dipole source via electric-field-driven LC resonator metamaterials, Phys. Rev. Lett. 111 (2013) 133901.
23. R. Yang, W. Tang, Y. Hao, A broadband zone plate lens from transformation optics, Optics Express 19 (2011) 12348.

24. O. Quevedo-Teruel, W. Tang, R.C. Mitchell-Thomas, A. Dyke, H. Dyke, L. Zhang, S. Haq, Y. Hao, Transformation optics for antennas: why limit the bandwidth with metamaterials, Sci. Rep. 3 (2013) 1903.

25. R. Yang, W. Tang, Y. Hao, Wideband beam-steerable flat reflectors via transformation optics, IEEE Antennas Wirel. Propag. Lett. 10 (2011) 1290.

26. J. Yi, S.N. Burokur, G.-P. Piau, A. de Lustrac, Restoring in-phase emissions from non-planar radiating elements using a transformation optics based lens, Appl. Phys. Lett. 107 (2015) 024101.

27. J. Yi, S.N. Burokur, G.-P. Piau, A. de Lustrac, Coherent beam control with an all-dielectric transformation optics based lens, Sci. Rep. 6 (2016) 18819.

28. COMSOL Multiphysics Modeling (http://www.comsol.com)

29. CST Microwave Studio (http://www.cst.com)

Cite this article as: Joshi C, Lepage AC \& Begaud X: A Dielectric-only superstrate inspired from transformation optics for complete reorientation of electromagnetic waves in azimuthal plane. EPJ Appl. Metamat. 2016, 3, 5. 